\title{
APLICACIONES ACTUALES DE LAS TECNICAS RADIOISOTOPICAS EN LA PATOLOGIA ENDOCRINOLOGICA
}

\author{
Dr. José A. Canessa G.
}

Servicios de Medicina Nuclear. Hospital de la Dirección de Previsión de Carabineros de Chile y Centro Radiológico Fleming. Santiago

\begin{abstract}
Nuclear Endocrinology was the first clinical use of radionuclides, five decades ago. From those days to the present, nuclear medicine has a definitive role in the diagnosis of a great variety of clinical situations, from the very frequent thyroid nodule to the unusual neuroendocrine tumors, including parathyroid hyperfunctioning tissue. Radioiodine $l^{131}$ still remains a very effective treatment for differentiated thyroid cancer and whole body scan with $I^{131}$ in conjunction with seric Tyroglobulin level are the main follow-up strategies for these tumors. New techniques, like positron emission tomography (PET) and newer radiolabeled peptides, among others, will offer a molecular approach to the $21^{\text {st }}$ century clinical nuclear medicine.
\end{abstract}

Key Words: Thyroid, Parathyroid, Adrenal gland, MIBG, MIBI, Technetium, Tumors.

Resumen: Se presentan las principales indicaciones actuales de los estudios radioisotópicos en endocrinología clínica, y su situación relativa con el resto de las técnicas de imagen no invasivas disponibles en nuestro medio. Se discuten brevemente las nuevas aplicaciones terapéuticas y su potencial desarrollo.

Palabras Claves: Tiroides, Paratiroides, Suprarrenal, Tumores neuroendocrinos, Tecnecio-99m, Yodo-131, Indio-111, MIBI, Octreoscan, MIBG, Feocromocitoma, Neuroblastoma, Tumores APUD, Hipercortisolismo, Hiperaldosteronismo, Terapéutica.

Introducción

La Medicina Nuclear se incorporó a la práctica clínica desde los laboratorios de endocrinología, siendo la patología tiroidea uno de sus primeros campos de aplicación diagnóstica y terapéutica. En Diciembre de 1946 se utilizó yodo-131 por primera vez en tratamiento de cáncer tiroideo metastásico por Seidlin, Martinelli y Oshry en el Hospital Montefiori de Nueva Cork, experiencia publicada en JAMA.

En 1951 Benedict Cassen construye el cintígrafo

Canessa JA. Aplicaciones actuales de las técnicas radioisotópicas en la patología endocrinológica. Rev Chil Radiol 2002; 8: 53-58.

Correspondencia: Dr. José A. Canessa G.

Vital Apoquindo 1200. Las Condes. Casilla 50090

e-mail: jose.canessa@hospitaldipreca.cl lineal, primer equipo para la obtención de imágenes derivadas de Medicina Nuclear, utilizando Yodo-131 y Oro-198.

Previamente, Emilio Segré, junto a Edmond Perrier, sintetizan el Tecnecio en 1937, primer elemento producido artificialmente por el hombre, bombardeando molibdeno con deuterones. El Tecnecio-99m es el radionúclido fundamental de la medicina nuclear actual, aun cuando su aplicación masiva pudo ser efectiva cuando se desarrolló la gama-cámara, por Hal Anger en $1956^{(1)}$.

En el amplio campo de la endocrinología clínica, las técnicas de medicina nuclear tienen indicaciones diagnósticas y terapéuticas.

\section{A. APLICACIONES DIAGNOSTICAS \\ A 1. Tiroides}

La patología nodular del tiroides es bastante común, con una prevalencia clínica del $4-7 \%$ y una incidencia anual del $0.1 \%$. De estos nódulos, el $4-5 \%$ son malignos. Los nódulos no palpables son aún más frecuentes, estimándose en 30-50\% la incidencia global, en series basadas en autopsia o como hallazgo incidental en estudios ultrasonográficos. La frecuencia es mayor en mujeres, y se incrementa con la edad, asociándose a deficiencia endémica de yodo e historia de exposición a radiaciones ionizantes ${ }^{(2-4)}$.

Los nódulos tiroideos deben estudiarse por su potencial de malignización, de hipertiroidismo o síntomas compresivos. Debido al importante desarrollo del ultrasonido y de la técnica de punción aspirativa con aguja fina, la cintigrafía tiroidea se complementa muy bien con éstas por su valor funcional.

\section{Técnica}

Actualmente se utiliza el Tecnecio-99m, cuyas características físicas son ideales para imágenes con gama-cámara y su comportamiento como anión es similar al yoduro, ya que es captado por la célula tiroidea por mecanismo de transporte activo, aun cuando no es organificado. A los 20-30 minutos de la inyección endovenosa de 5-10 mCi de pertecneciato de sodio, se obtienen imágenes del cuello en proyecciones frontales y oblicuas, con marcas de referencia en horquilla esternal y mentón. Por consideraciones dosimétricas (dosis de 
radiación elevada), el uso de Yodo-131 se desaconseja. En nuestro medio no contamos con Yodo123 , cuyas características físicas y químicas son ideales para imágenes. Las imágenes con Tecnecio-99m tienen muy buena correlación con Yodo-131, se ha descrito un $5-8 \%$ de discordancia consistente en nódulo funcionante con tecnecio y no funcionante con Yodo, aún cuando esta aparente discordancia no tendría impacto clínico, ya que en la serie de Kusic ninguno de estos casos correspondió a una neoplasia tiroidea ${ }^{(5,6)}$.

\section{Indicaciones}

La principal indicación actual en la evaluación del tiroides nodular es la presencia de niveles circulantes disminuidos de hormona tiroestimulante (TSH), ya que puede corresponder a un nódulo funcionante. El resto de la patología nodular, con niveles de TSH normales o altos es evaluada más eficientemente con ecotomografía de alta resolución la que puede guiar una punción aspirativa con aguja fina (PAAF) (Figuras 1 y 2).

Otras indicaciones, menos frecuentes de la cintigrafía tiroidea son: 1) Evaluación de masa cervical, para descartar tejido tiroideo ectópico funcionante que se puede encontrar en el quiste tirogloso y lóbulo piramidal, entre otros, 2) Localización de tejido tiroideo remanente post cirugía, 3) Localización de tiroides retroesternal, 4) Evaluación de bocio doloroso (tiroiditis aguda), 5) Evaluación de tiroiditis crónica de Hashimoto $^{(5)}$.

La cintigrafía tiroidea puede ser complementada con la determinación de la captación tiroidea porcentual de una dosis mínima de Yodo-131, la que se efectúa a las 2, 6 y/o 24 horas de su administración oral y permite una estimación del nivel de función glandular global. La captación tiroidea de radioyodo, como parámetro de función tiroidea, ha sido reemplazada en gran parte de sus indicaciones por las determinaciones mucho más precisas de niveles hormonales (T3, T4, TSH y sus fracciones) y actualmente sus indicaciones están restringidas a la confirmación de una tiroiditis aguda, la evaluación de respuesta nodular a la terapia de supresión con hormona tiroidea exógena y la determinación de masa glandular residual post tiroidectomía y con fines de cálculo de dosis terapéutica de radioyodo.

En el cáncer diferenciado del tiroides la exploración corporal con radioyodo mantiene plena vigencia diagnóstica. Se efectúa a las 72 a 96 horas de la administración de una dosis baja de Yodo-131 (3-5 mCi, vía oral), obteniéndose imágenes de cuerpo entero, lo que permite detectar pequeñas cantidades de tejido tirodeo normotópico sea residual o recidivado y tejido metastásico. Para una adecuada captación del radioyodo se requieren niveles elevados de TSH (sobre $30 \mathrm{mU} / \mathrm{ml}$ ), lo que se obtiene suspendiendo la terapia de reemplazo hormonal al menos 30 días antes del examen, en el caso de sustitución con Eutirox ${ }^{\circledR}$, o con el

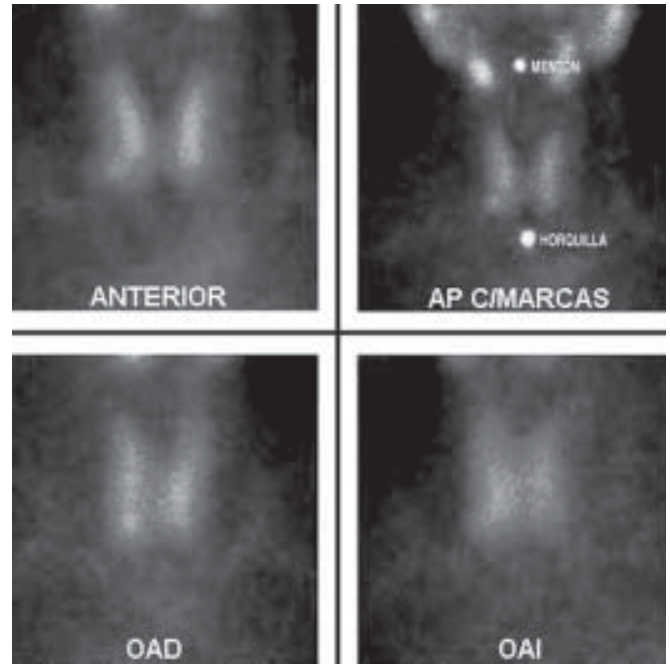

Figura 1 Cintigrafía de tiroides con Tecnecio-99m. Proyecciones anterior, anterior con marcas de referencia en horquilla esternal y mentón y proyecciones oblicuas. Aumento de tamaño del tiroides y leve irregularidad de captación en el polo inferior del lóbulo derecho, compatible con bocio difuso de grado moderado.

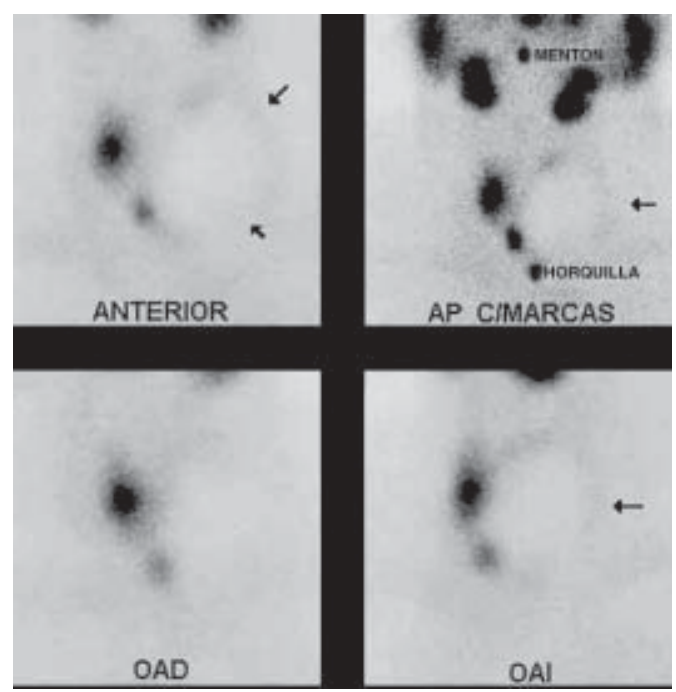

Figura 2. Cintigrafía de tiroides con Tecnecio-99m Proyecciones anterior, anterior con marcas de referencia en horquilla esternal y mentón y proyecciones oblicuas. Marcado aumento de tamaño del lóbulo tiroideo izquierdo, correspondiente a un gran nódulo (flechas) que no concentra el Tecnecio-99m y que corresponde a un nódulo quístico en la ecotomografía.

uso de TSH recombinante (rTSH, Thyrogen ${ }^{\circledR}$ ), lo que evita los molestos síntomas de hipotiroidismo que presentan los pacientes. La TSH recombinante no está disponible en nuestro medio y su costo es bastante elevado(7).

La exploración corporal con yodo o "rastreo sistémico", tiene una elevada sensibilidad en la detección de recidiva de cáncer tiroideo diferenciado y se correlaciona con el aumento de niveles de Tiroglobulina (Tg) (Figuras 3 y 4). Un tema aún en discusión es la discordancia, poco frecuente, entre el resultado de la 


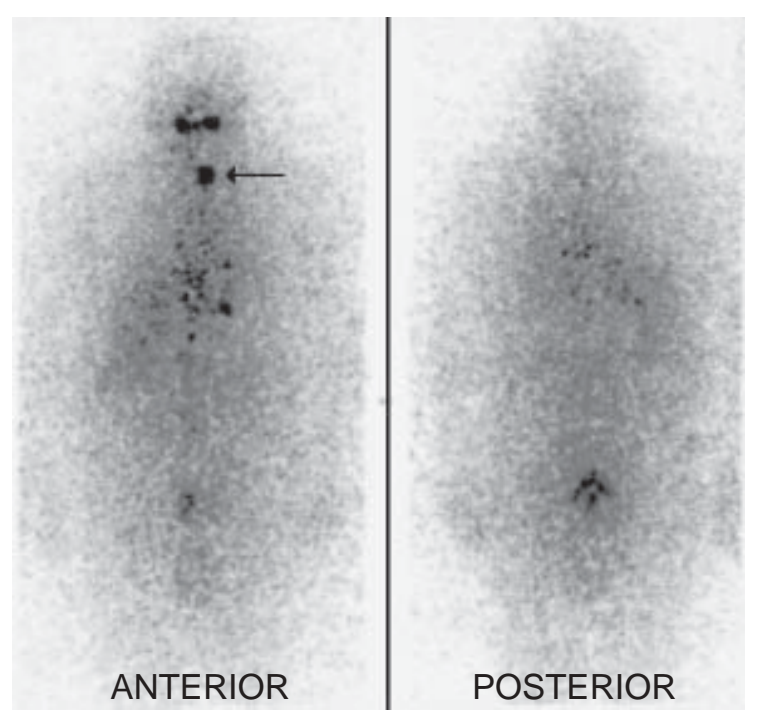

Figura 3. Exploración corporal con Radioyodo-131. Imagen de cuerpo entero en proyección anterior y posterior, a los siete días de la administración terapéutica de $200 \mathrm{mCi}$ (7,4 $\mathrm{GBq}$ ) de $\mathrm{l}^{131}$, por un cáncer diferenciado del tiroides. Tiroidectomía total seis semanas antes. Se aprecia captación del yodo-131 en región cervical anterior, en relación con remanente tiroideo post quirúrgico.

exploración corporal y los niveles de Tg. En el caso de exploración positiva con Tg negativa, deben descartarse causas conocidas de falso negativo para Tg, la más frecuente es la presencia de auto-anticuerpos anti-Tg. El caso contrario es más difícil de evaluar. Niveles elevados de $\mathrm{Tg}$ con rastreo corporal negativo requiere buscar tejido tiroideo metastásico que ha perdido la capacidad de captar radioyodo, con otras técnicas de imágenes. El uso de una dosis ablativa de yodo-131 en forma empírica en estos casos es aún discutible ${ }^{(8)}$.

El Yodo-131 tiene un papel terapéutico esencial en el manejo del cáncer diferenciado del tiroides, lo que discutiremos más adelante. A la semana o 10 días de la administración de una dosis terapéutica de yodo-131 es posible efectuar una exploración corporal, la que al utilizar una dosis 10-20 veces superior puede detectar entre $10-30 \%$ de nuevos focos metastáticos ${ }^{(9)}$.

\section{A 2. Paratiroides}

Debido a su pequeño tamaño (40 mg de peso) las glándulas paratiroides son de difícil evaluación no invasiva. Se ubican frecuentemente en relación con los polos tiroideos, aun cuando pueden estar también en mediastino y región cervical inferior.

Inicialmente se dispuso de técnicas de sustracción de imágenes con Talio-201 y Tecnecio-99m, lo que fue superado por la introducción a fines de la década de 1980 de los isonitrilos, de los cuales el metoxi-isobutilisonitrilo (MIBI) es el que tiene más aplicación clínica. Marcado con Tecnecio-99m, presenta un comportamiento farmacocinético particular; inicialmente es captado tanto por células tiroideas como paratiroideas y posteriormente permanece por más tiempo en las células paratiroideas. Por lo tanto, la obtención de

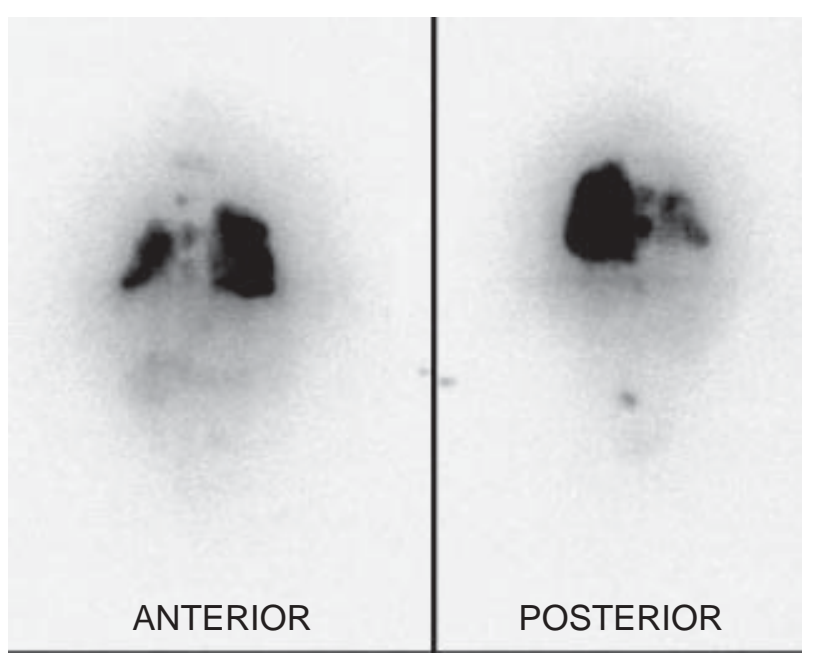

Figura 4. Exploración corporal con Radioyodo-131. Imagen de cuerpo entero en proyección anterior y posterior, a los 7 días de la administración terapéutica de 200 mCi (7,4 GBq) de $l^{131}$, por un cáncer diferenciado del tiroides (Tiroidectomía total seis semanas antes). Intensa captación anormal del radioyodo en ambos campos pulmonares, mayor a izquierda, consistente con diseminación micronodular pulmonar bilateral.

imágenes precoces (20 minutos post inyección) y tardías (2 horas) permiten demostrar claramente la captación de MIBI en glándulas paratiroides hiperfuncionantes 0 aumentadas de tamaño. Ayuda en la interpretación una imagen de cintigrafía tiroidea con tecnecio(10) (Figura 5) . El método es especialmente sensible para la localización de adenomas y tiene menor rendimiento en glándulas hiperplásicas. Las imágenes con MIBI son superiores al resto de las técnicas no invasivas, incluyendo ecotomografía de alta resolución, tomografía axial (TAC) y resonancia magnética (RM) y es claramente superior a la antigua técnica de doble isótopo (Talio-Tecnecio) con sustracción digital. La técnica tiene algunos falsos positivos, en general nódulos tiroideos que concentran MIBI y está indicada en la evaluación de estados hipercalcémicos en los que se sospeche un hiperparatiroidismo y casos de elevación de paratohormona (PTH). De especial interés es la capacidad de las imágenes con MIBI de explorar no sólo el cuello, sino también el mediastino superior.

\section{A 3. Glándula suprarrenal}

\section{A 3 a. Corteza suprarrenal}

En la corteza suprarrenal se sintetizan hormonas esteroidales, utilizando como precursor el colesterol. Los análogos de colesterol marcados con Yodo-131, especialmente el 6-beta-iodometil-19-norcolesterol (Norchol $\left.{ }^{\circledR}, \mathrm{NP}-59\right)$ permiten, al ser transportado en la sangre unido a lipoproteínas de baja densidad (LDL), unirse a receptores celulares de la corteza suprarrenal con esterificación intracelular. Esto posibilita localizar tumores funcionantes productores de hormonas, como cortisol (Síndrome de Cushing), aldosterona 
a

\section{Minutos}

(Enfermedad de Conn, entre otras), dehidroepiandrosterona y androstenediona (síndromes de hiperandrogenismo adrenal)(11) y evaluar tumores no funcionantes que en general son encontrados como hallazgo en estudio de imágenes no invasivas por otra causa (incidentalomas). Esto último no es infrecuente, alcanzando un $1-10 \%$ de incidencia en estudio con TAC (12).

La técnica de imagen consiste en la inyección endovenosa de 0.5 -1.0 mCi de Yodo-131-NP-59, previa protección tiroidea con lugol y la obtención de imágenes de abdomen a los 5-10 días, tiempo que requiere el NP-59 para incorporarse al pool de colesterol y alcanzar la corteza suprarrenal. Dependiendo de la situación clínica, puede efectuarse prueba de supresión con dexametasona para frenar la actividad de ACTH hipofisiaria (2 mg vía oral al día, desde siete días antes de la inyección de NP-59 hasta el término de la secuencia de imágenes) $)^{(11)}$.

Las imágenes con NP-59 deben correlacionarse con la clínica, marcadores bioquímicos pertinentes y otros estudios de imágenes para una adecuada interpretación.

En síndrome de Cushing, la captación de NP-59 en ambas cortezas suprarrenales, simétrica, apunta a hipercortisolismo dependiente de ACTH, los que son de origen hipotalámico, hipofisiario o producto de síntesis ectópica de ACTH. En cambio, la captación bilateral pero asimétrica orienta a un hipercortisolismo independiente de $\mathrm{ACTH}$, cuya causa más frecuente es la hiperplasia nodular cortical. El contraste unilateral obliga a descartar un adenoma cortical suprarrenal. La ausencia bilateral de captación es de interpretación más incierta, asociada a carcinoma adrenal(13).

En hiperaldosteronismo primario, especialmente con supresión con dexametasona, la sensibilidad y especificidad de las imágenes con NP-59 es de $89 \%$ y $87 \%$, respectivamente ${ }^{(13)}$. En este caso tiene valor diagnóstico la precocidad del contraste cortical: Contraste unilateral precoz, que es el que ocurre antes de cinco días post-inyección, sugiere adenoma o carcinoma, en cambio contraste bilateral precoz orienta hacia hiperplasia cortical. En hiperaldos-teronismo secundario hay poca experiencia. b

Figura 5 a,b. Cintigrafía de Paratiroides con Isonitrilo (MIBI)-Tecnecio-99m a: Proyección anterior a los 20 min. $y$ dos horas de la inyección de MIBI. b: Imagen con Tecnecio-99m para localización del tiroides. Se observa un nódulo hipercaptante, especialmente en las imágenes de dos horas (flecha), en relación con el polo inferior del lóbulo tiroideo derecho, correspondiente a un nódulo paratiroideo hiperfuncionante.

Los estados de hiperandrogenismo de origen suprarrenal corresponden a adenomas o carcinomas unilaterales o más frecuentemente a hiperplasia bilateral secundaria a ovario poliquístico(14).

En el incidentaloma suprarrenal, como hallazgo de imágenes de resonancia magnética o tomografía axial, el NP-59 permite evaluar el estado funcional del nódulo: Captación de NP-59 en el nódulo incidental apunta a un origen benigno, sobre todo en tumores mayores a 1.5$2.0 \mathrm{~cm}$. de diámetro. Una masa que no capta NP-59 amerita mayor estudio, incluyendo biopsia o seguimiento estrecho con imágenes ${ }^{(11,15)}$.

\section{A 3 b. Medula suprarrenal}

La médula suprarrenal tiene funciones neuroendocrinas, especialmente la producción de catecolaminas. El uso de análogos iodados de guanetidina, como la Meta-yodo-bencil-guanidina (MIBG) marcada con Yodo-131 o idealmente con Yodo123 permite evaluarla, ya que MIBG es captado y concentrado en neuronas y tejido neuro-endocrino del sistema simpático-medular.

Previa protección tiroidea con lugol, se inyecta vía endovenosa 0.5-1.0 mCi de MIBG-I-131 ó 3-10 mCi de MIBG-I-123, obteniéndose imágenes del primer al tercer día post-inyección. Puede ser útil inyectar un trazador de excreción renal, como DTPA-Tecnecio-99m para localizar topográficamente el riñón. De estar disponible, las imágenes con Yodo-123 permiten un estudio tomográfico (SPECT), lo que aumenta el rendimiento de la técnica.

Las principales indicaciones son la localización de feocromocitomas y neuroblastomas. Los feocromocitomas son productores de epinefrina o norepinefrina, en general son unilaterales y benignos, aun cuando un $10 \%$ son bilaterales y un $2-10 \%$ son malignos. Pueden formar parte de neoplasias endocrinas múltiples (NEM) u otros cuadros hereditarios e infrecuentes. La MIBG tiene elevada sensibilidad y especificidad de $86 \%$ y $99 \%$, en series de más de 1900 pacientes $^{(16)}$. La especificidad de MIBG es superior al de TAC y RM, aún cuando estos últimos tienen mayor sensibilidad y se prefiere iniciar con estas técnicas el estudio del paciente ${ }^{(17)}$. 
Los neuroblastomas afectan a niños menores de cinco años y pueden producir norepinefrina, dopamina o L-dopa. La MIBG detecta el tumor primario y sus metástasis con sensibilidad sobre $90 \%$, cifras similares son obtenidas con RM, que tiene la ventaja de no usar radiación ionizante, por lo que es preferida en población pediátrica $^{(18)}$.

\section{A 4. Tumores neuroendocrinos}

En los últimos años se ha incorporado al arsenal diagnóstico un análogo estructural de somatostatina, el Octreotide marcado con Indio-111 (Octreoscan ${ }^{\circledR}$ ). La somatostatina es un péptido de 14 aminoácidos con acción inhibitoria de la hormona de crecimiento. Diversas estirpes celulares expresan receptores de somatostatina en su membrana, entre las que destacan células neuroendocrinas derivadas de la cresta neural. Estos grupos celulares altamente especializados se conocieron inicialmente como células APUD ("Amine Precursor Uptake and Decarboxilation"). Este tejido es productor de hormonas peptídicas y aminas neurotransmisoras. El tejido APUD actualmente se clasifica como "neuroendocrino"(19). Los tumores neuroendocrinos más frecuentes son: tumores carcinoides, gastrinomas, glucagonomas, insulinomas, paragangliomas y vipomas. También se incluyen el carcinoma medular del tiroides y el tumor pulmonar de células pequeñas.

Las imágenes con Octreoscan se obtienen mediante la inyección endovenosa de 4-6 mCi de Indio111 Octreotide, con estudio de cuerpo entero a las 4 y 24 horas y eventualmente estudio tomográfico (SPECT) (Figura 6). La sensibilidad global del método es de 79\% para localización tumoral. El rendimiento más bajo es para insulinomas. Octreoscan parece ser más sensible que MIBG en carcinoide, cáncer medular del tiroides y tumores de los islotes del páncreas y MIBG es superior en feocrocitomas y neuroblastomas ${ }^{(20-24)}$.

El cáncer medular de tiroides puede ser evaluado con ácido dimetil-mercapto-succínico pentavalente (DMSA-V) marcado con Tecnecio-99m, MIBG y Octreoscan, con resultados variables. Su uso en todo caso es de segunda línea diagnóstica(25).

\section{B. APLICACIONES TERAPEUTICAS}

El aspecto más conocido es el uso de radioyodo (Yodo-131) en el tratamiento del hipertiroidismo y del cáncer diferenciado del tiroides. Se basa en la emisión beta del Yodo-131, de alta energía y corto alcance, la que, al ser incorporada a la célula tiroidea, produce su destrucción sin mayor daño sistémico.

En hipertiroidismo se utiliza cuando ha fracasado el uso de propiltiouracilo (PTU), o como alternativa a la cirugía tiroidea. Se administra vía oral una dosis de 5$30 \mathrm{mCi}$, dependiendo del tamaño glandular, la severidad del hipertiroidismo y la presencia o no de nódulos funcionantes. Los nódulos tóxicos son más

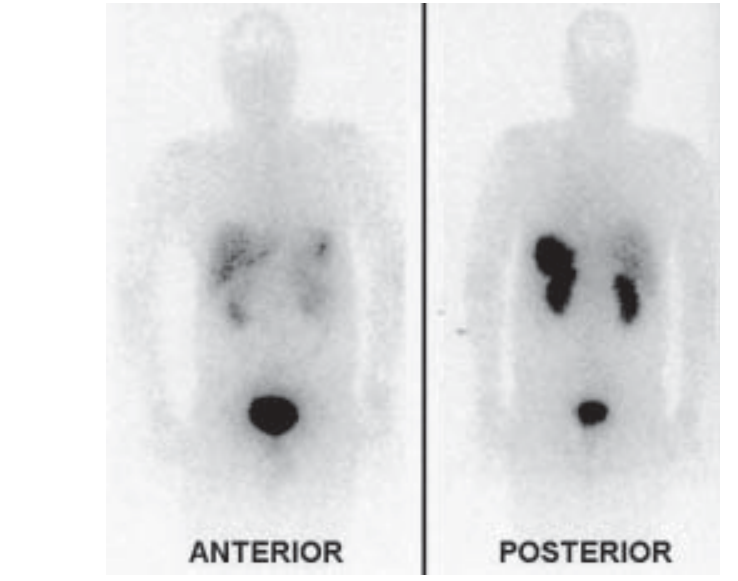

Figura 6. Exploración corporal con Octreotide (Octreoscan) Indio-111. Imagen de cuerpo entero anterior y posterior a las 24 horas de la inyección de Octreoscan.

Estudio normal. Captación normal en hígado y bazo y excreción renal.

radioresistentes, por lo que requieren una dosis mayor.

Existen diferentes algoritmos de cálculo de dosis a administrar, la mayoría engorrosos y poco prácticos, por lo que en general se administra una dosis fija corregida por la captación glandular de 24 horas y la masa tiroidea estimada. El procedimiento es en general bien tolerado y aún cuando el ideal es llevar al paciente a un estado de eutiroidismo, la alta tasa de fracaso obliga más bien a llevar a hipotiroidismo y proceder posteriormente a sustitución hormonal. La elección de radioyodo o cirugía sub-total es una opción del equipo de trabajo de cada centro dedicado al tema, ya que ambas opciones tienen ventajas y desventajas.

En el cáncer diferenciado del tiroides la administración de una dosis ablativa de Yodo-131, complementaria a la cirugía con criterio oncológico, es fundamental en la sobrevida y calidad de vida del paciente. A las 4-6 semanas de la tiroidectomía total, tiempo necesario para que se eleven los niveles de TSH sobre $30 \mathrm{mU} / \mathrm{ml}$, se administran entre 100-200 mCi de Yodo-131. Aún cuando el tratamiento es muy bien tolerado, se requiere aislar al paciente por 48-96 horas, para evitar irradiación inadvertida a población general(26). Los efectos secundarios son menores y autolimitados (sialoadenitis, depresión medular transitoria, irritación gástrica, alteración del gusto). No se ha reportado aumento de incidencia de otros tumores, aún con dosis muy altas (800 $\mathrm{mCi})$.

La ablación tiroidea total permite disponer del marcador plasmático tiroglobulina $(\mathrm{Tg})$ y de la exploración sistémica con Yodo-131 como valiosos elementos en el seguimiento clínico de estos pacientes ${ }^{(5)}$.

Recientemente se han incorporado otros radiofármacos al campo terapéutico de los tumores neuroendocrinos. Son los mismos agentes de imagen, administrados en dosis mayores. MIBG-Yodo-131 se ha utilizado en tratamiento de feocromocitomas, neuroblastomas, tumores carcinoides, paragangliomas y cáncer 
medular del tiroides. Los mejores resultados se han obtenido en feocromocitoma agresivo, con respuesta en al menos un $55 \%$ de los casos ${ }^{(27,28)}$

Menos experiencia está disponible en pacientes tratados con análogos de somatostatina (OctreoscanIndio-111) en dosis alta (150-500 mCi), con buenos resultados y sin efectos secundarios severos ${ }^{(27)}$. Este es un campo atractivo y con potencial desarrollo futuro.

Finalmente, no se puede dejar de mencionar el gran campo que la técnica de tomografía por emisión de positrones (PET), desarrollada en otro apartado de este número, tiene en el campo endocrinológico, en especial en neoplasias hormono-dependientes, especialmente prolactinomas ${ }^{(29)}$ y cáncer diferenciado de tiroides con imágenes negativas con Yodo-131(30). Es indudable que las técnicas PET dominarán el futuro de las imágenes de Medicina Nuclear, abriendo un campo de investigación y diagnóstico a nivel molecular, de alcances insospechados.

\section{Bibliografia}

1. Canessa JA, Abarzúa E. Breve historia de las radiaciones y su aplicación en Medicina Nuclear: Un homenaje a Wilhelm Roentgen y George Von Hevesy. Rev Chilena de Radiología. 1996; 2: 12- 17

2. Cases JA, Surks MI. The changing role of scintigraphy in the evaluation of thyroid nodules. Semin Nucl Med 2000; 30: 81-87

3. Gharib $\mathrm{H}$. Changing concepts in the diagnosis and management of thyroid nodules. Endocrinol Metab Clin North Am 1997; 26:777-800

4. Rojeski MT, Gharib H. Nodular thyroid disease. Evaluation and management. N Eng J Med 1985; 313: 428-436

5. Dworkin HJ, Meier DA, Kaplan M. Advances in the management of patients with thyroid disease. Semin Nucl Med 1995; 25: 205-220

6. Kusic Z, Becker DV, Saenger E.L. y col. Comparison of technetium-99m and iodine-123 imaging of thyroid nodules: Correlation with pathologic findings. J Nucl Med 1990; 31: 393-399

7. Ladenson P.W. Recombinant thyrotropin versus thyroid hormone withdrawal in evaluating patients with thyroid carcinoma. Semin Nucl Med 2000; 30: 98-106

8. Fatourechi V, Hay ID. Treating the patient with differentiated thyroid cancer with thyroglobulin-positive iodine-131 diagnostic scan - negative metastases: Including comments on the role of serum thyroglobulin monitoring in tumor surveillance. Semin Nucl Med. 2000; 30: 107-114

9. Sherman SI, Tielens ET, Sostre S. y col. Clinical utility of posttreatment radioiodine scans in the management of patients with thyroid carcinoma. J Clin Endocrinol Metab 1994; 78: 629-634

10. McBiles M, Lambert A, Cote MG, Kim SY. Sestamibi parathyroid imaging. Semin Nucl Med 1995; 25: 221-234

11. Freitas JE. Adrenal cortical and medullary imaging. Semin Nucl Med 1995; 25: 235-250

12. Copeland PM. The incidentally discovered adrenal mass. Ann Intern Med 1983; 98: 940-945

13. Gross MD, Shapiro B, Grekin RJ y col. Scintigraphic localization of adrenal lesions in primary aldosteronism. Am J Med 1984; 77: 839-844

14. Givens JR. Hirsutism and hyperandrogenism. Adv Intern Med 1976; 21: 221-247

15. Gross MD, Shapiro B, Francis IR y col. Scintigraphic evaluation of clinically silent adrenal masses. J Nucl Med 1994 ; $35: 1145-1152$

16. Campeau RJ, García OM, Correa OA y col. Pheocromocytoma: Diagnosis by scintigraphy using 131-metaiodobenzylguanidine. South Med J. 1991; 84: 1221-1230

17. Chatal JF. Can we agree on the best imaging procedure(s) for localization of pheocromocytoma?. J Nucl Med 1993; 34: 180-181

18. Hoefnagel CA, Voute PA, de Kraker y col. Radionuclide diagnosis and therapy of neural crest tumors using I131 metaiodobenzylguanidine. J Nucl Med 1987; 28: 308-314

19. Olsen JO, Pozderaqc RV, Hinkle G y col. Somatostatin receptor imaging of neuroendocrine tumors with indium111 pentreotide (Octreoscan) Semin Nucl Med 1995; 25: 251-261

20. Lamberts WJ, Krenning EP, Reubi JC y col. The role of somatostatin and its analogs in the diagnosis and treatment of tumors. Endocr Rev 1991; 12: 450-482

21. Krenning EP, Kwekkeboom DJ, Bakker WH y col. Somatostatin receptor scintigraphy with $\left({ }^{111} \mathrm{In}-\mathrm{DTPA}-\mathrm{D}-\right.$ Phe) and (123-Try3) octreotide : The Rotterdam experience with more than 1000 patients. Eur J Nucl Med 1993; 20: 716-731

22. King CMP, Rezhek RH, Bomanji J y col. Imaging neuroendocrine tumors with radiolabeled somatostatin analogues and $\mathrm{x}$-ray computed tomography: A comparative study. Clin Radiol 1993; 48: 386-397

23. Kaltsas G, Korbonits E, Heintz J y col. Comparison of somatostatin analog and metaiodobenzylguanidine radionuclides in the diagnosis and localization of advanced neuroendocrine tumors. J Clin Endocrinol Metab 2001; 86: 895- 902

24. Brandi ML, Gagel RF, Angeli A y col. Consensus : Guidelines for diagnosis and therapy of MEN type 1 and type 2. J Clin Endocrinol Metab 2001; 86: 56585657

25. Clarke SEM, Lazarus $C R$, Wraight $P$ y col. Pentavalent -99m Tc DMSA, ${ }^{131}$ I MIBG and Tc ${ }^{99 m}$ MDP. An evaluation of three imaging techniques in patients with medullary carcinoma of the thyroid. J Nucl Med 1988; 29: 33-38

26. Canessa JA. Algunas consideraciones prácticas sobre radioprotección en Medicina Nuclear. Rev Chil Radiol 1996; 2: 27-32

27. Wiseman GA, Kvols LK. Therapy of neuroendocrine tumors with radiolabeled MIBG and somatostatine analogues. Semin Nucl Med 1995; 25: 272-278

28. Troncone L, Galli G. Proceedings of the international workshop on the role of I $^{131}$ MIBG in the treatment of neural crest tumors. J Nucl Biol Med 1991; 35: 177-362

29. De Landsheere C, Lamotte D. Methods and clinical applications of positron emission tomography in endocrinology. Ann Endocrinol (Paris) 1990; 51: 148-154

30. Krausz Y. Nuclear endocrinology as a monitoring tool. Semin Nucl Med 2001; 31: 238-250 